\title{
Nature of Black Holes and Space-Time around Them
}

\author{
Amir Ali Tavajoh \\ Adib Astronomy Center, Esfahan, Iran \\ Email: Amir_ali3640@yahoo.com
}

How to cite this paper: Tavajoh, A.A. (2017) Nature of Black Holes and SpaceTime around Them. Journal of High Energy Physics, Gravitation and Cosmology, 3, 96-105.

http://dx.doi.org/10.4236/jhepgc.2017.31013

Received: September 25, 2016

Accepted: December 25, 2016

Published: December 29, 2016

Copyright $\odot 2017$ by author and Scientific Research Publishing Inc. This work is licensed under the Creative Commons Attribution International License (CC BY 4.0).

http://creativecommons.org/licenses/by/4.0/

\begin{abstract}
Based on the Mach's principle, black holes warp the space time in a way that geodesic for every object which is moving toward black hole starts to bend and object starts to rotate around the black hole. Even light cannot be able to escape from the strong gravitational field of black hole and all the light like paths will warp so as to fall farther to the hole. Before arriving to the Schwarzschild's Sphere, object faces with length extension because of the difference between amount of tidal forces on the nearest and furthest points of object that take the object apart and after passing the Schwarzschild's sphere, based on the Special relativity of Einstein, the parts of object face with length contraction. In comparison between strange stars and black holes we conclude that core of strange stars has a temperature and pressure not sufficient for up and down quarks and they turn into strange ones. However, in core of black holes, because of massive stars and hot gases falling into it, they are always in a high temperature and pressure. So they can be made up of up and down quarks. At the Ergo sphere Region of black hole, a particle that gets into it will divide into 2 pieces, one of them falls into the black hole and another gets out of the Schwarzschild sphere very fast and it's called the black hole radiation. According to the Diagram drawn by R. Rafini and J. Weeler, an object gets out of white hole in past space-time, it can be able to send signals to us and we can receive it but black hole which is located in future space-time, after object enters to the Schwarzschild's Sphere, the signals it sends won't be received. In order to reach the third space-time which is like a mirror to our universe, our speed needs to exceed the speed of light to pass the Einstein-Rosen Bridge. As a conclusion, structure of black holes can be made up of up and down quarks and everything falls into the black hole, collapses and turns into a bunch of quarks. Space-time around black holes, based on Rafini-Weeler diagram, is like a frontier between our space-time and other space-times. So it can be possible to reach past space-time and other space-times.
\end{abstract}

\section{Keywords}

General Relativity, Geodesic, Rafini-Weeler Diagram, Strange Stars, Einstein-Rosen Bridge, Schwarzschild's Sphere, Ergo Sphere, Space-Time Curvature, Time Warp, Special Relativity, Mach’s Principle 


\section{Introduction}

Have you ever noticed when water swirl around the drain?

In 1783, John Michell, rector and leading scientist in the village of Thornhill, suggested that surface of gravity of some stars could be so strong that even light cannot be able to escape from them.

In 1915, Albert Einstein pointed out that gravity is a warp in space time caused by matter on general relativity.

The more massive an object, the greater it warps the space around it.

In 1916, German astronomer, Karl Schwarzschild found that enough matter packed into small enough space, would have such a powerful gravitational field that nothing can escape from it.

In 1963, Roy Kerr showed that massive stars will drag the space time around them like the water swirling around the drain.

His equations were just specified for black holes.

In 1963, Maurten Schmidt discovers an odd star-like point of light which right now we call them "Quasar". This discovery was leaded to the realization that all the quasars are powered by super massive black holes.

In 1967, John A. Weeler, officially named "black hole" for these collapsed stars [1].

A particular type of star is called "Quark Star" or "strange star" which means that their core is made up of strange quarks and their shell is made up of degenerate neutrons.

The reason why strange quarks are fundamentals of quark star's core rather than up and down quarks which are fundamentals of neutrons is that up and down quarks require ultra-high pressure and temperature which a neutron star cannot provide that much high level of temperature and pressure so they can be strange quarks [2] [3]. These stars, because of their density, are very similar to the black holes.

Possible structure of black holes and comparison between black holes and quark stars, will be discussed in this paper.

\section{General Properties of Black Holes}

\subsection{How Stars Turn into Black Hole}

Based on General relativity of Albert Einstein, there's no "gravitational force" and the term "gravitational field" is used instead.

Also based on Mach's principle, matter tells geometry how to curve and geometry tells matter how to move [4].

Black holes are the remains from super massive stars after supernova. Please notice that singularity is another word for Black Holes.

When the star collapses, the density goes to infinity. The more massive a star, the more it warps the space time and curves geodesic of an object falling into it if and only if the mass of the object coming toward it is less than the star's mass. (Geodesic is the shortest distance between two points in space which is not always a straight line and if the two points are located in gravitational fields it will be a curve rather than a straight line) [5]. 
Only stars whose mass is over Tolman-Oppenheimer-Volkoff limit (3 solar masses) can transform into black hole [6].

The gravitational field of black holes are so strong that even light cannot be able to escape from them.

\subsection{Types and Structure of Black Holes}

Black holes are made up of 2 parts:

1) Event horizon: A well-defined surface that nothing can be seen and escape, because the required escape velocity exceeds velocity of light [7]. It's known as point of no return [8].

In other word, within this horizon, all light like paths, and hence all paths in the forward light cones of particles within the horizon, are warped so as to fall farther into the hole (see Figure 1) [9] [10].

Based on Doppler Effect, objects while approaching to the event horizon have red shift in their spectrum because they're getting further from us and closer to the black hole so fast [11], also because they come from the upper gravitational potential (far from the black hole) to the lower gravitational potential (event horizon), they emit $\mathrm{X}$-rays before arriving to the event horizon. Black holes are categorized in group of $\mathrm{X}$-ray binaries as the same as pulsars [12].

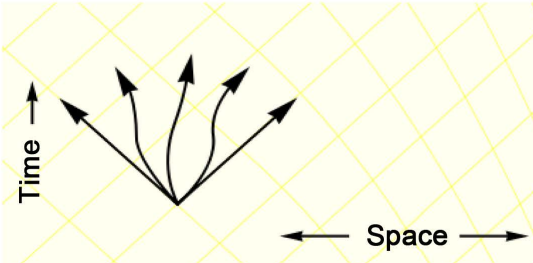

(a)

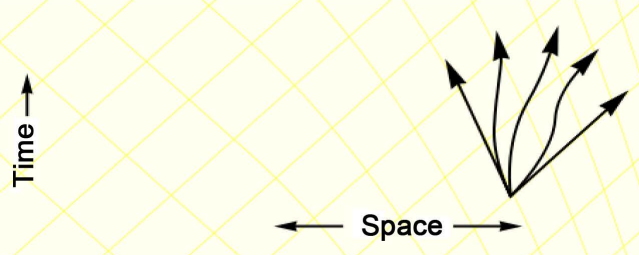

(b)
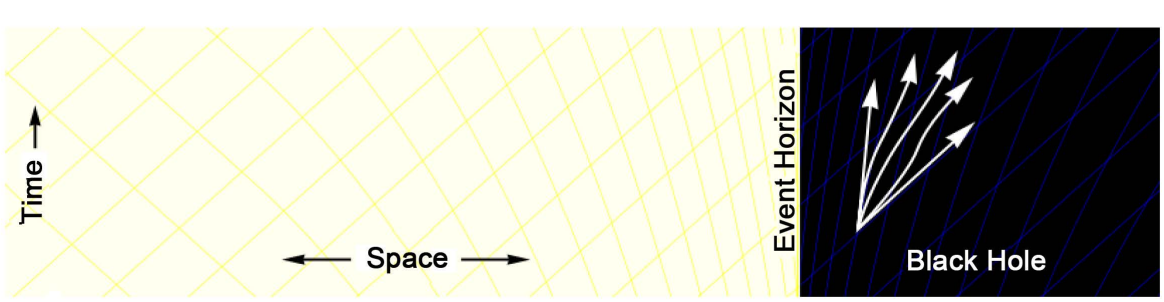

(c)

Figure 1. (a) far away from black hole, a particle can move in any direction, it's only restricted by the velocity of light; (b) closer to the black hole, space time starts to deform, in some convenient coordinate systems, there are more paths going toward the black hole than paths moving away; (c) inside the event horizon, all the paths bring the particle close to the center of black hole. It's no longer possible for the particle to escape. 
2) Black hole's cone: knowledge we have about this part of black hole is based on mathematical equations. Please note that the term "cone" is just the geodesic of matter going through black hole or in another word, it's the hypothetical shape of space-time near the black hole in 3 dimensions rather than 4 dimensions( 3 dimensions of space and 1 dimension of time) because we are unable to imagine 4 dimensions.

Karl Schwarzschild, German Astronomer in 1916, found a formula for obtaining the radius of sphere around black hole that if any object enters into this sphere, it cannot be able to escape anymore and also if the object emits signal toward us, signal cannot be able to escape from strong gravitational field either.

Event Horizon is Schwarzschild's radius.

Schwarzschild's radius can be obtained by the following formula:

$$
R_{\mathrm{s}}=\frac{2 G m}{C^{2}}
$$

\subsection{Types of Black Holes}

There are 3 types of black holes:

1. Black holes located at the center of galaxies; this type of black holes are extremely massive and they can be able to hold the stars and interstellar gases around themselves.

2. Normal Black holes; this type of black holes is approximately impossible to find because they are like a trap for beams of light, except some rare situations such as Cygnus X-1. Cygnus X-1 was the first time astronomers could find a black hole which is beside a red giant star. This is a source of X-ray because gases move from upper to lower gravitational potential and produce X-ray. [13]

3. Rotational black holes; In contrast to what we believe, black holes emit particles. When supernova takes place, star starts to collapse and based on angular momentum conservation, the rotation of star needs to increase until it turns to black hole and the poles of star get closer to each other, equator gets bigger and black hole's shape is shown in Figure 2. When a particle comes into the Ergo sphere, it turns into 2 pieces; one of them falls into the black hole and another, gets out of black hole very fast and it can be detected. Energy of the second particle, comes from the energy transferred from rotational black hole to the particle [14].

Because of this, Stephen Hawking, in 1974, showed that black holes are not black and they emit a form of radiation.

\subsection{Effects of Black Hole's Gravitational Field and Black Hole's Fundamental Particles}

When an object approaches the Schwarzschild's sphere, because of the difference between amount of gravitational field on nearest and furthest points of object and also tidal forces acting on it, cause length extension and the object turns apart into pieces (Figure 3) [15].

After the pieces of object goes through Schwarzschild's sphere, every piece of it faces with relativistic length contraction because based on angular momentum conservation, as the distance between pieces of an object and the center of black hole diminishes, the 


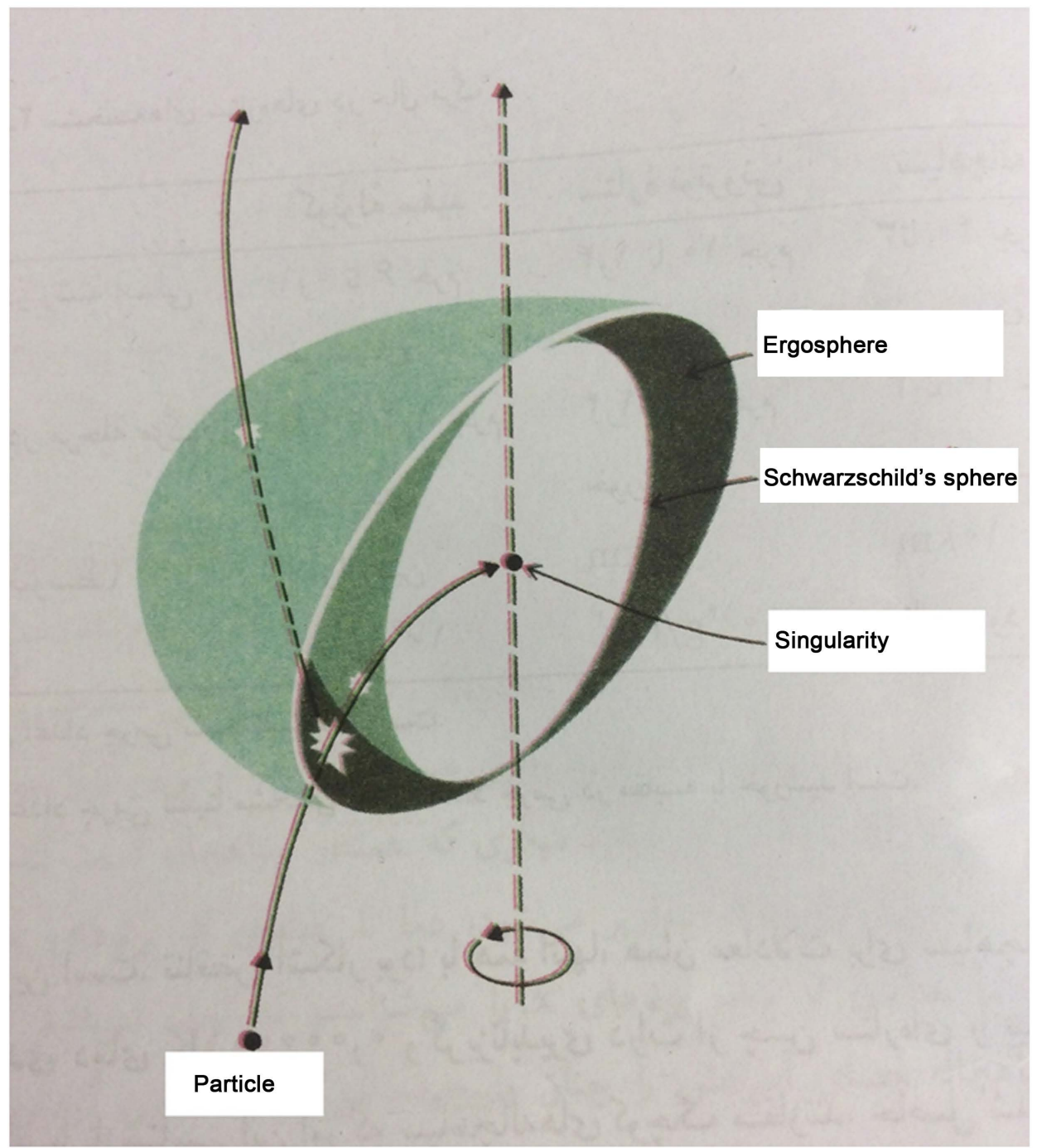

Figure 2. When a particle comes into the black hole, in Ergo sphere, it turns into 2 pieces and one of them goes through Schwarzschild's sphere and another gets out of black hole very faster than the first particle.

velocity of pieces of object gets higher and higher until it approaches to the speed of light [16].

$$
L=m(R \times v)
$$

So the based on relativistic length contraction we have:

$$
L^{\prime}=L\left(1-\frac{v^{2}}{c^{2}}\right)^{\frac{1}{2}}
$$

So it can be possible that the pieces of object have some changes in their structure, such as neutron stars that when gases in star will be under strong pressure the electrons fall into the core of atom and interact with protons and make neutrons.

So if the collapse process goes on, the neutrons would break into their fundamentals which are two $-1 / 3$ quarks and one $2 / 3$ quark.

Black holes can be made up of quarks. 


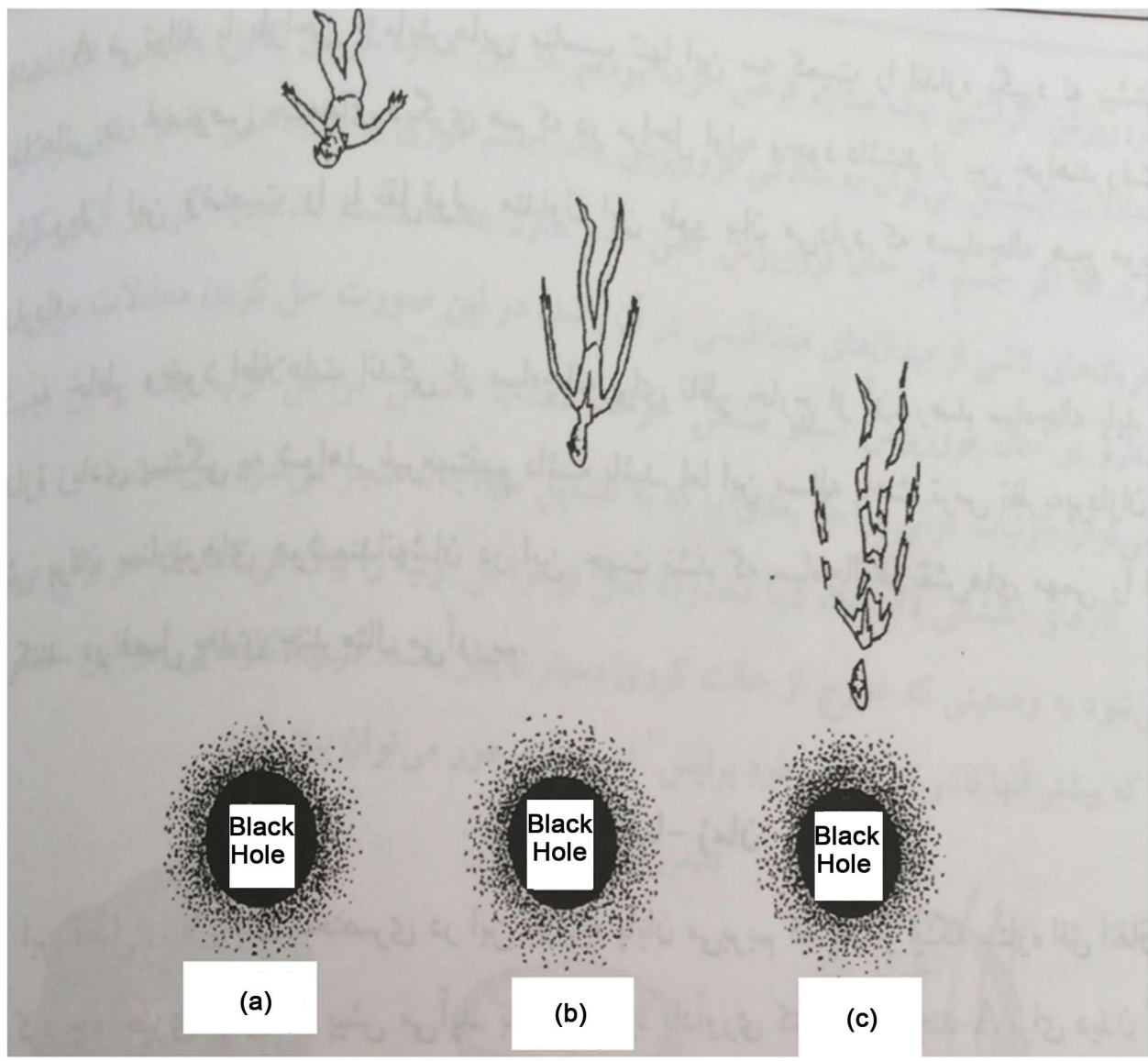

Figure 3. (a) The object or human far from black hole; (b) when it approaches to the black hole; (c) when its position is so close to the Schwarzschild's sphere.

Quark stars are made up of strange quark and degenerate neutron (strange quarks located in the core and degenerate neutrons located in the shell). Core of quark stars is made up of Strange quarks because there is no strong pressure acting on them. However, at first, core of quark stars were "up" and "down" but these quarks have Fermi energy and they are very unstable at low pressure and temperatures. So they transform into Strange quarks. Quark stars are often called "strange stars".

In comparison between strange stars and black holes, there are differences such as:

1. Strange stars have always the same amount of mass and it doesn't increase, in contrast to them, everything falls into the black hole and its mass always is increasing. So, as the amount of mass increases, amount of pressure acting on the core of black hole increases as well and up and down quarks can be stable.

2. Most of the times stars and hot interstellar gases fall into the black hole, so they increase the temperature of black hole and up and down quarks of the core of black hole remain stable.

First, because everything in the nature needs to get along with the nature's properties. So, if the fundamentals of black hole are quarks, everything falls into black hole, collapses and turns into a cocktail of quarks. And second, at that much high pressure and temperature, degenerate neutrons located at the shell, also cannot remain stable and decay into up and down quarks. 


\section{Space-Time around Black Holes}

\section{Rafini-Weeler Diagram and Worm Holes}

R. Rafini and J. Weeler, 2 scientists on 1967, represented the space-time near black hole in a diagram (Diagram 1 ).

Imagine an Astronomer and his friend are going to have an experiment, astronomer wants to go through black hole and his friend stay away from black hole and take signals astronomer sends. Before astronomer arrives to the Schwarzschild's radius, he sends signals to his friend and his friend receive them without any problem (points A and B in Diagram 1) and his friend is traveling from past to the future in the space shuttle (line $O$ in Diagram 1) But when astronomer enters to the Schwarzschild's sphere, no signal will be delivered by his friend (points C, D, E and F in Diagram 1).

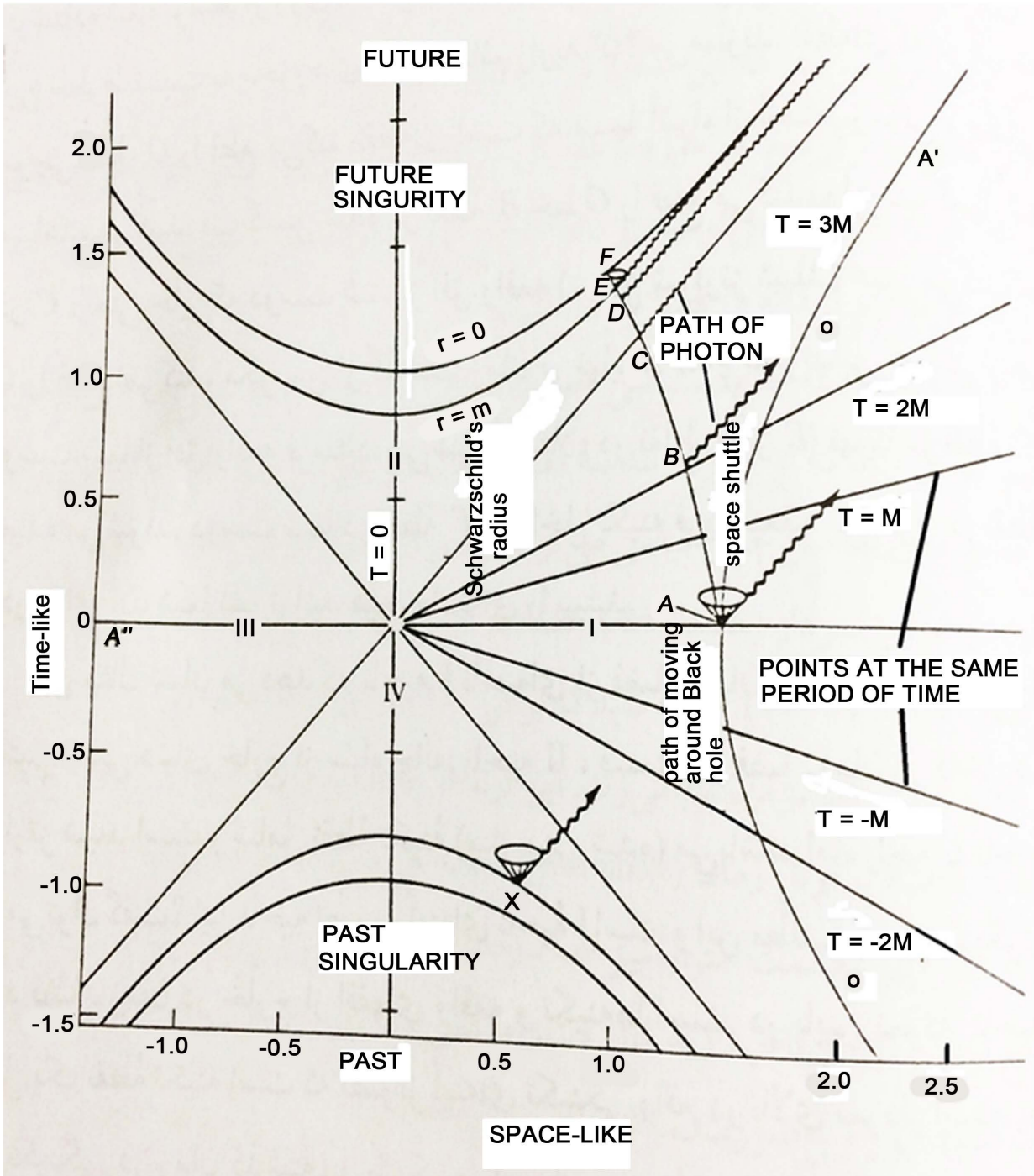

Diagram 1. The space time near black hole. Astronomer approaches to the black hole (point A to F) and sends signals to his friend who's moving on line $O$ in the Space Shuttle and receives astronomer's signals. Also when astronomer gets out of the white hole in past space-time on point $\mathrm{X}$, he can be able to send signals to his friend and his friend can deliver them without any problem. 
In Diagram 1, there are 4 space-times; first one is our universe's space-time. The second one is the future space-time. Third one is like a mirror of our universe; we have no idea about this universe because it's unreachable except one condition.

We can be able to reach that universe through Einstein-Rosen Bridge or in other word, worm hole. Although, worm hole just opens in a short period of time. During this period, we need to go through it with a velocity more than the velocity of light (Figure 4).

Worm holes are bridges located between black holes and white holes and the parameters of space time inside and outside the worm hole are very different; imagine we have a timer inside and outside the worm hole, the difference between our measurements is shown in Figure 5. It's similar to the relativistic time dilation in special relativity but there is a difference which is the object travels back in time [17].

Fourth one is the past space-time. Black holes have convergence and everything around them will fall into them by their strong gravitational field. But in this spacetime, in contrast to black holes, we have divergence for objects which have already been fallen into black hole and they are called "White Hole". As you see in Diagram 1, the astronomer that gets out of white hole, can send signals from point $\mathrm{X}$ to his friend without any problem and he will deliver it [18].

\section{Conclusions}

1) It's probable that black holes made up of up and down quarks.

2) In order to reach third world (take a look at Diagram 1) worm holes, integrals between connection between black hole and white hole, connect us to that space-time which is past space-time.

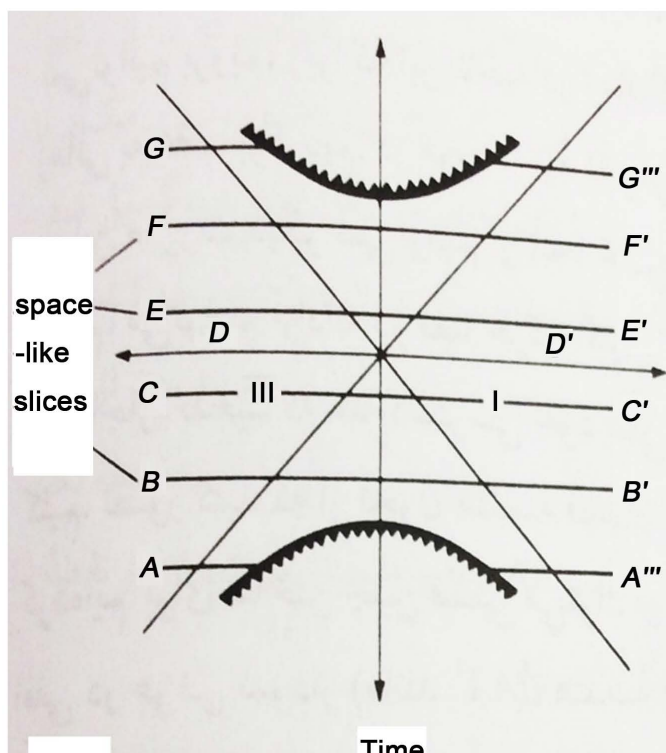

(a)
Time

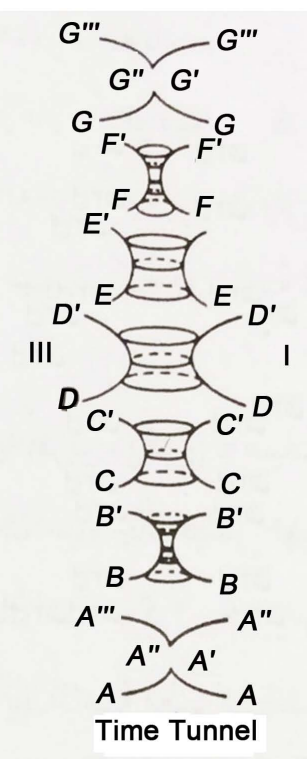

(b)

Figure 4. Space-time evolutions near black hole in order to create worm holes. (a) Space-time hypothetical slices from various periods of time; (b) geometry specified for each space-time slice. We can see the time tunnel which opens and closes very quickly (From A to G). 


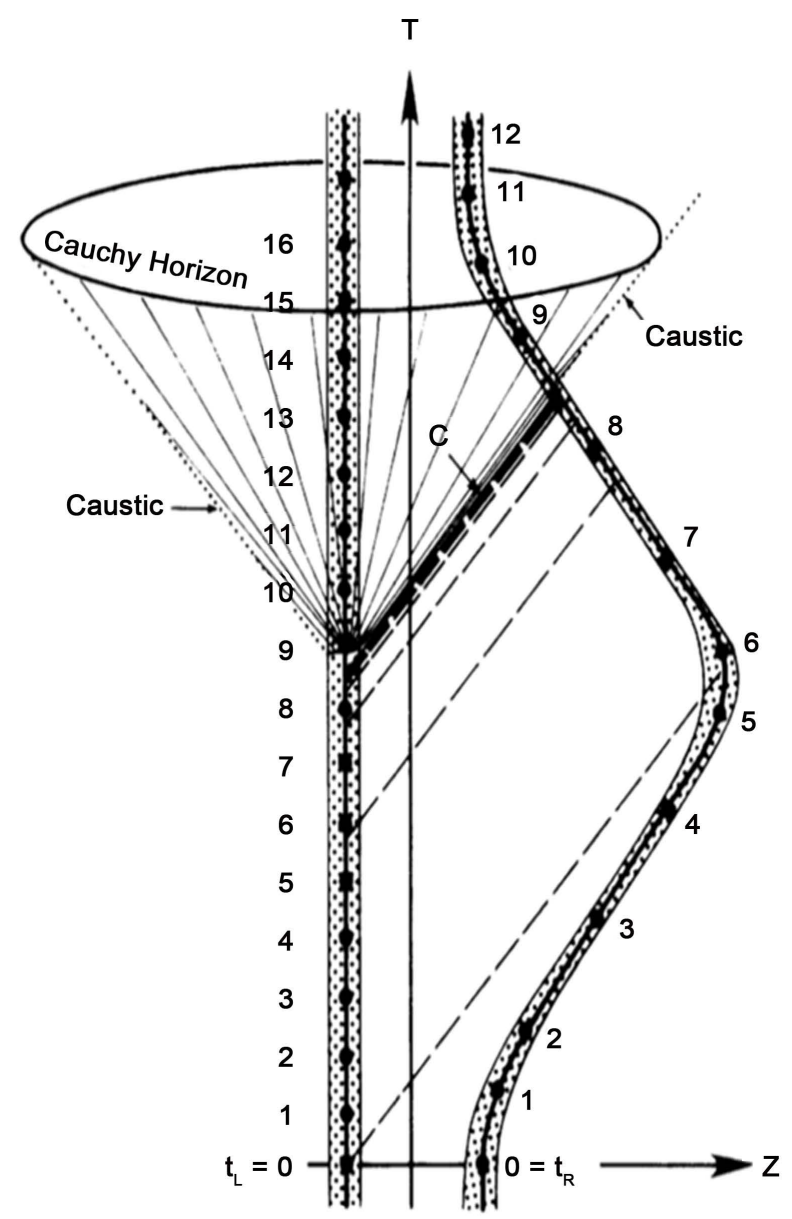

Figure 5. The difference between measurements inside and outside the worm hole; $t_{L}$ is the timer outside the worm hole and $t_{R}$ is the timer inside the worm hole. $\mathrm{C}$ is the velocity of light that shows time-like curve at the mouth of worm hole. $\mathrm{T}$ is the time coordinate and $\mathrm{Z}$ is space coordinate.

Also, the objects already fell into the black hole, get out of the white hole in the past space time and based on Rafini-Weeler diagram, and they can reach our space-time (by sending signals).

On the other hand, based on Rafini-weeler diagram, when object falls into the black hole and passes Schwarzschild's sphere, it moves to the future Space-Time and we should notice that black holes are located in our space-time too.

So, black holes are the frontier between all space-times.

\section{References}

[1] http://blackholes.stardate.org/history.html

[2] Ivanenko, D.D. and Kurdgelaize, D.F. (1965) Hypothesis Concerning Quark Stars. Astrophysics, 1, 251-252. https://doi.org/10.1007/BF01042830

[3] Ivanenko, D.D. and Kurdgelaize, D.F. (1969) Remarks on Quark Stars. Lettere Al NuovoCimento Journal, Volume 2, Number 1.

[4] Gron, O. and Naess, A. (2011) Einstein's Theory. Springer, Oslo.

[5] Ellis, G. and Williams, R. (1988) Flat and Curved Space-Times. Clarendon Press, Oxford. 
[6] http://en.m.wikipedia.org/wiki/Event_horizon

[7] http://www.physicsoftheuniverse.com/topics_blackholes_event.html

[8] Hester, J., Smith, B., Blumenthal, G., Kay, L. and Voss, H. (2010) 21st Century Astronomy. 3rd Edition, W.W. Norton \& Company, New York.

[9] Margalef-Bentabol, B., Margalef-Bentabol, J. and Cepa, J. (2012) Evolution of the Cosmological Horizons in a Concordance Universe. Journal of Astroparticle Physics and Cosmology, 2012.

[10] Margalef-Bentabol, B., Margalef-Bentabol, J. and Cepa, J. (2013) Evolution of the Cosmological Horizons in a Universe with Countably Infinitely Many State Equations. Journal of Cosmology and Astroparticle Physics, 2013. https://doi.org/10.1088/1475-7516/2013/02/015

[11] Rindler, W. (1977) Essential Relativity: General, Special and Cosmological. 2nd Edition, Springer-Verlag, New York.

[12] http://www.novacelestia.com/space_art_binary/X-ray_binaries.html

[13] http://www.nasa.gov/mission_pages/galex/galex20120502.html

[14] Dixon, R.T. (1992) Dynamic Astronomy. 6th Edition, Prentice-Hall, Prentice-Hall, Eaglewood Cliffs, New Jersy.

[15] Narlikar, J.V. (1996) Lighter Side of Gravity. 2nd Edition, Cambridge University Press, New York. https://doi.org/10.1017/CBO9780511600258

[16] Resnick, R. (1972) Introduction to Special Relativity. Wiley Fastern Private Limited, New York.

[17] Morris, M., Thorne, K. and Yurtsever, U. (1988) Wormholes, Time Machines, and the Weak Energy Condition. Physical Review Letters, 61, 1446-1449. https://doi.org/10.1103/PhysRevLett.61.1446

[18] Zeilik, M. and Smith, E.V.P. (1997) Introductory Astronomy and Astrophysics. 4th Edition, Second Volume, Wiley, New York.

Submit or recommend next manuscript to SCIRP and we will provide best service for you:

Accepting pre-submission inquiries through Email, Facebook, LinkedIn, Twitter, etc. A wide selection of journals (inclusive of 9 subjects, more than 200 journals)

Providing 24-hour high-quality service

User-friendly online submission system

Fair and swift peer-review system

Efficient typesetting and proofreading procedure

Display of the result of downloads and visits, as well as the number of cited articles

Maximum dissemination of your research work

Submit your manuscript at: http://papersubmission.scirp.org/

Or contact jhepgc@scirp.org 